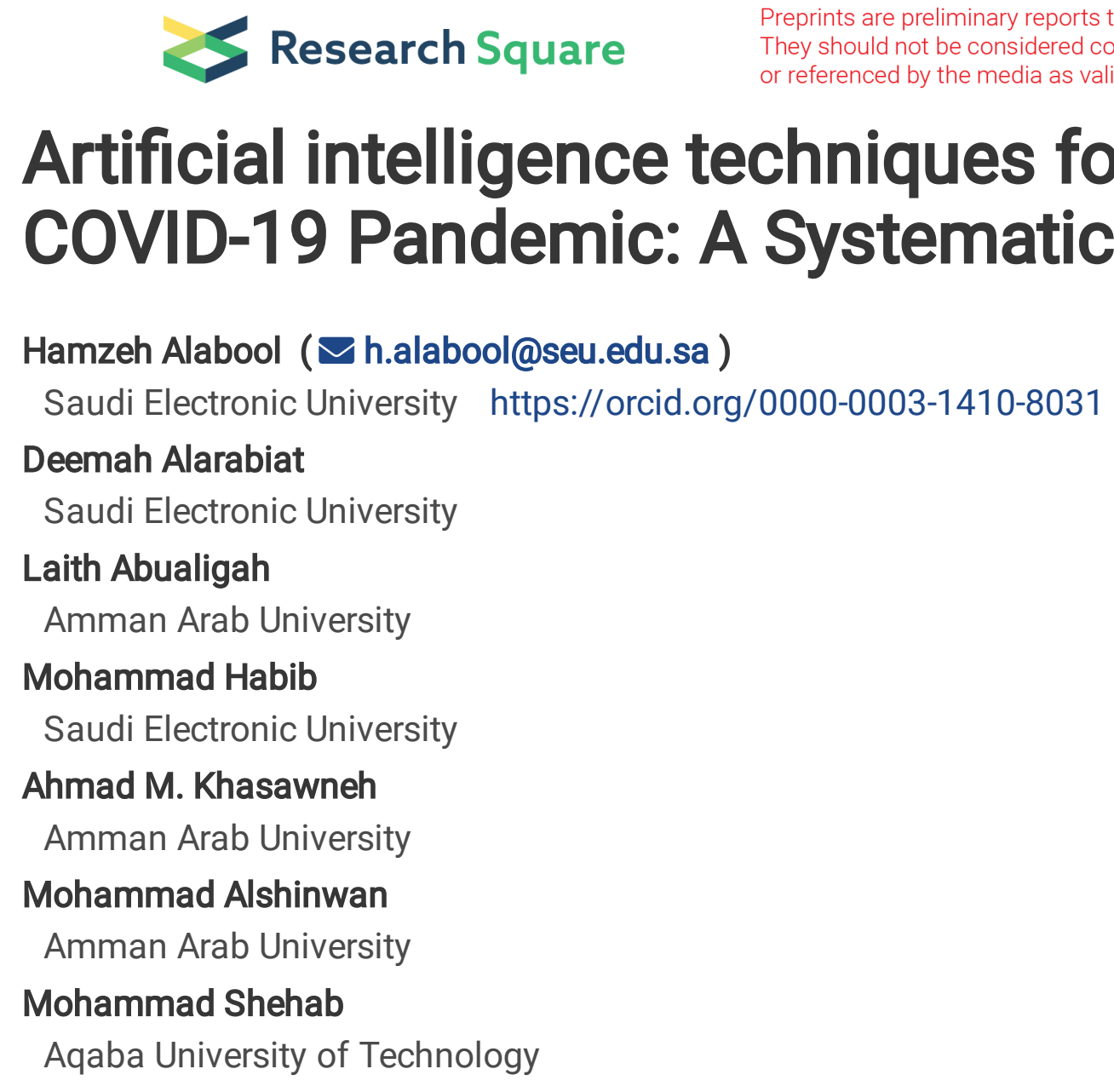

\author{
Hamzeh Alabool ( $\sim$ h.alabool@seu.edu.sa ) \\ Saudi Electronic University https://orcid.org/0000-0003-1410-8031 \\ Deemah Alarabiat \\ Saudi Electronic University \\ Laith Abualigah \\ Amman Arab University \\ Mohammad Habib \\ Saudi Electronic University \\ Ahmad M. Khasawneh \\ Amman Arab University \\ Mohammad Alshinwan \\ Amman Arab University \\ Mohammad Shehab \\ Aqaba University of Technology
}

\title{
Artificial intelligence techniques for Containment COVID-19 Pandemic: A Systematic Review
}

\section{Systematic Review}

Keywords: COVID-19, Coronavirus, SARS-CoV-2, Artificial Intelligence, Healthcare, Review

Posted Date: May 21st, 2020

DOI: https://doi.org/10.21203/rs.3.rs-30432/v1

License: (c) (i) This work is licensed under a Creative Commons Attribution 4.0 International License.

Read Full License 


\section{Abstract}

Due to the advantages offered by Al in containment the COVID-19 pandemic, the number of Al techniques has increased greatly. Although these techniques provide an acceptable start to COVID-19 pandemic control, they differ in terms of purpose, Al synthesis methods, datasets, validation approach. This increase and diversity in the numbers of proposed Al techniques can confuse decision makers and lead them to the dilemma of what is the appropriate technique under the specific conditions. Yet, studies that assess, analyze, and summarize the unresolved problems and shortcomings of current Al techniques for COVID-19 are limited. In the existing review studies, only individual parts of Al techniques, rarely the full solution, are reviewed and examined. Thus, this study aims to present a comprehensive systematic review on the application of Al techniques in containment the COVID-19 pandemic. The applied search strategy led to include 73 papers related to the Application of Al techniques for COVID-19 published from December 2019 to May 2020. Ten applications of Al for containment COVID-19 were identified. In addition, the analysis results of the systematic review revealed five deficiencies so that future research should take them into consideration.

\section{Introduction}

The first status recorded for COVID-19 disease was identified on December 2019 in Wuhan, China. By the end of January 2020, there were almost 12,000 recorded cases in China. In the middle of March 2020, the World Health Organization declared the COVID-19 to be a pandemic due to the fact of spreading it globally within a short time period (around 203 countries) (WHO, 2020). Till 10th of May, there were $4,168,125$ confirmed COVID-19 cases in a total of 210 countries that had resulted in 1,467,407 recovered and 283,189 deaths (Worldmeater, 2020).

As a reaction to the COVID-19 pandemic, the rapid growth of the literature review that offered different solutions in controlling and monitoring the growth of COVID-19 pandemics has been noticed. For instance, the support of existing technologies (e.g., Big Data, Internet of Things and Artificial Intelligence) are investigated to help prevent the spread of the virus by predicting high-risk patients, tracking previous infected cases and analyzing previous data of the patients (Vaishya et al., 2020) ensured that Al is a potentially powerful technology that can be utilized to provide sophisticated solutions to overcome the COVID-19 pandemic. Al is used in different ways and can play a significant role in the Big Data in the terms of pattern recognition, explanation and prediction. These functions can be useful to diagnose, predict, and treat COVID-19 infections. (Fuchs 2020) stated that Al can contribute against COVID-19 at main six areas: "early warnings and alerts, tracking and prediction, data dashboards, diagnosis and prognosis, treatments, and cures, and social control". Al can be utilized to find complex relationships in a large set of data in a short period of time. Moreover, Al-based models can be used to observe more data about patients such as side effects of different cures based on patients predefined records; this can help to identify the most suitable management approach to be used for each patient and help to speed up the process of finding a suitable vaccine for the COVID-19 disease. 
Therefore, due to the advantages offered by Al in containment the COVID-19 pandemic, the number of Al techniques has increased greatly. Although these techniques provide an acceptable start to COVID-19 pandemic control, they differ in terms of purpose, Al synthesis methods, data collection techniques, datasets, validation approach. This increase and diversity in the numbers of proposed Al techniques can confuse decision makers and lead them to the dilemma of what is the appropriate technique under the specific conditions. Thus, this study aims to present a comprehensive systematic review on the application of Al techniques in containment the COVID- 19 pandemic. This study is significant due to its advantages of identifying and categorizing the applications of Al techniques in containment the COVID19 pandemic, compare and summarize the he deficiencies of Al techniques in containment the COVID-19 pandemic. The article is organized into five sections which are:

- Research Background

- Related Works

- Methodology

- Descriptive analysis and discussion of the review results

- Discussion of future research directions in light of the findings

- Validity and Reliability of Research Findings

\section{Related Work}

In Table 1. Eleven review studies related to the applications of Al in COVID-19 pandemic have been identified and summarized. The majority of these studies are conducted to identify the possible applications of AI for COCID-19 pandemic. While, some of these review studies conducted to review the applications of Al techniques for (1) detecting covid-19 from chest X-ray images (2) classification medical imaging for COVID-19, managing of COVID-19 ICU patients, and critically appraise prediction models for covid-19. The details of these review studies are discussed as follows:

Vaishya et al., (2020) presented a short review study aims to identify the possible applications of Al for COVID-19 pandemic. Although the study indicated the search sources (e.g., ubmed, Scopus and Google Scholar) and Keywords (e.g., COVID-19 or Coronavirus and Artificial Intelligence or Al) that were used in the process of retrieving relevant research, the studies used in the review process were not disclosed. This review concluded that seven areas in which Al can be applied to confront the COVID-19 pandemic, which are: (1) Early detection and diagnosis of the infection (2) Monitoring the treatment (3) Contact tracing of the individuals (4) Projection of cases and mortality (5) Development of drugs and vaccines (6) Reducing the workload of health care workers (7) Prevention of the disease.

Naudé (2020) conducted a review study to assess the role of artificial intelligence in areas in which it could be used to counter the COVID-19 pandemic. These areas such as (1) early warnings and alerts, (2) tracking and prediction, (3) data dashboards, (4) diagnosis and prognosis, (5) treatments and cures, and 
(6) social control. The study concludes that the lack of data and by too much noisy and outlier data; Al will not be effective in confronting the COVID-19 pandemic.

The study released by the Center for Hypertension and Precision Medicine and Clinical Pharmacology, William Harvey Research Institute (Alimadadi et al., 2020) identified the possible application of Al for COVID-19 pandemic. The study revealed that Al cloud be used to (1) understand the pattern of viral spread (2) improve diagnostic speed and accuracy (3) develop novel effective therapeutic approaches (4) identify the most susceptible people based on personalized genetic and physiological characteristics. The study recommended that as the technology of Al depends heavily on the size of data in the accuracy of its outputs, it is necessary to develop a huge and centralized COVID-19 patient database for scientific purposes.

Nguyen (2020) Reviewed 17 papers that are related to the application of Al for COVID-19 Pandemic. The study reported that Al can be used in many areas in the fighting of COVID-19 pandemic. These areas such as (1) medical image processing (2) computational biology and Medicine (3) COVID-19 infection rate forecasting (4) COVID-19 Modelling (5) COVID-19 detection (6) COVID-19 drug discovery.

llyas et al., (2020) reviewed five papers related to the application of Al for detecting COVID-19 from chest X-ray images. The study concluded that AI technology cannot be relied upon to detect COVID-19 disease using X-ray images. This is because pneumonia can be caused by many other viruses except for COVID19. Therefore, the study advised that if artificial intelligence is applied in this field, it is necessary that the study be based on much more data size than 100 X-ray images.

Van der Schaar, \& Alaa, (2020) conduct a review study to identify the challenges of healthcare systems respond to COVID-19. The study aims to show how artificial intelligence can be applied to solve the identified challenges. The review result identified five major challenges of healthcare systems respond to COVID-19 which are (1) managing limited healthcare resources (2) developing personalized patient management and treatment plan (3) informing policies and enabling effective collaboration (4) understanding and accounting for uncertainty (5) expediting clinical trials. To address these challenges that study suggests that implementation of proposed Al-based methods can be accomplished through three levels of human-machine interface which are (1) Individual-level interface (2) Hospital-level interface (3) Nation-level interface.

Table 1. Summary of the review studies on the applications of AI for COVID-19 pandemic. 


\begin{tabular}{|c|c|c|}
\hline Study & Focus Area & Study Objective \\
\hline $\begin{array}{l}\text { Vaishya et al., } \\
\text { (2020) }\end{array}$ & COVID-19 pandemic & $\begin{array}{l}\text { To identify the possible applications of AI for } \\
\text { COVID-19 pandemic }\end{array}$ \\
\hline \multirow[t]{2}{*}{ Naudé (2020) } & COVID-19 pandemic & To review the actual and potential contribution of $\mathrm{AI}$ \\
\hline & & to the fight against COVID-19 \\
\hline Alimadadi et al., & COVID-19 pandemic & To identify the possible applications of AI for \\
\hline (2020) & & COVID-19 pandemic \\
\hline \multirow[t]{2}{*}{ Nguyen (2020) } & COVID-19 pandemic & To identify the possible applications of AI for \\
\hline & & COVID-19 pandemic \\
\hline \multirow[t]{2}{*}{ Ilyas et al., (2020) } & Detecting Covid-19 From & To review approaches used for the detection of \\
\hline & Chest X-ray Images & COVID-19 \\
\hline van der Schaar, \& & COVID-19 pandemic & To identify the possible applications of AI for \\
\hline Alaa, (2020) & & COVID-19 pandemic \\
\hline Bullock et al., & COVID-19 pandemic & To review the actual and potential contribution of AI \\
\hline (2020) & & to the fight against COVID-19 \\
\hline \multirow[t]{2}{*}{ Shi et al., (2020) } & Medical imaging for COVID- & To review the possible application of AI to medical \\
\hline & 19 & imaging for COVID-19 \\
\hline Rahmatizadeh et & Critical care of the COVID-19 & To assess the AI role in the management of COVID- \\
\hline al., (2020) & patients & 19 ICU patients. \\
\hline \multirow[t]{2}{*}{ Latif et al., (2020) } & COVID-19 pandemic & To identify the possible Applications of data science \\
\hline & & for COVID-19 \\
\hline Wynants et al., & Diagnosis and prognosis of & To review and critically appraise prediction models \\
\hline$(2020)$ & covid-19 infection & for covid-19. \\
\hline
\end{tabular}

Bullock et al., (2020) presented a review study to identify the possible application of Al for COVID-19 pandemic. The study identified five scales that Al could be used to address the COVID- 19 challenges, namely patient, molecular, societal, and datasets and resources scales. Patient scale included 3 categories which are: (1) medical imaging for diagnosis (2) non-invasive measurements for disease tracking (3) protein structure prediction. While, molecular scale included (1) protein structure prediction (2) improving viral and testing (3) drug repurposing (4) drug discovery. Besides, societal scale which includes epidemiology and infodemiology. Lastly, datasets and resources scale which include case data, textual data and biomedical data.

Shi et al., (2020) reviewed the application of Al in imaging data acquisition, segmentation and diagnosis for COVID-19. The study demonstrated the role of artificial intelligence in assisting visualization technicians (e.g., X-ray and/or CT) in imaging facilities without direct contact with the patient. Also, the study reviewed the possibility of using Al for image segmentation and assisted diagnosis. The study recommended that in order to improve the results of Al techniques that applied for imaging data acquisition, segmentation and diagnosis for COVID-19 and make them clinically useful, the quality and number of COVID-19 patient data need to be further improved.

Rahmatizadeh et al., (2020) presented a review study that aims to identify the applications of Al techniques for critical care of the COVID-19 patients. Papers under review were retrieved from PubMed, Web of Science, Google scholar. The study proposed a three layers model for Al application in ICU for COVID-19 patients. 
Latif et al., (2020) reviewed the applications of data science for COVID-19 pandemic. The review aimed to identify the most significant challenges of COVID-19 and how data science can be used to address these challenges. Also, the study presented details of the most available datasets and resources of COVID-19 patient that could be useful for further research in this area. In addition, the study reviewed several studies that are related to image analysis, textual data mining, audio analysis, and embedded sensing. The review results shown that the most important data science challenges for COVID-19 pandemic relate to (1) Data Limitations (2) Correctness of Results (3) challenges related to Security, Privacy, and Ethics (4) The Need For Multidisciplinary Collaboration (5) The Need For Multidisciplinary Collaboration (6) New Data Modalities (7) Solutions for the Developing World.

Wynants et al., (2020) conducted a systematic review and critical appraisal existing prediction models for COVID-19 infection. Search sources such as PubMed and EMBASE are used to retrieve related studies. Total of Twenty-seven studies describing 31 prediction models were selected for the systematic review. The study concluded that all proposed models have good predictive performance, but they are high risk due to many deficiencies, such as poor reporting and poor methodological conduct for participant selection, predictor description, and statistical methods used.

As shown in Table 1. and the presented discussion of current reviews, a comprehensive systematic review of the recent Al techniques that applied for containment COVID-19 pandemic is lack. Seldom is the complete picture reviewed; only portions of the applications of Al techniques have been typically reviewed and examined. This systematic review covers a greater number of Al techniques for COVID-19, where there are many studies that still need to be investigated. Therefore, this review seeks to unify recent research efforts to help in strengthening new insights in pursuing more research that could improve on current Al techniques outputs or help to develop new Al techniques with better performance and accuracy for containment COVID-19 pandemic.

\section{Methodology}

The review methodology used in this study follows the guidelines and procedures proposed by (Keele, 2007; Kitchenham et al., 2009). The current review study is conducted from the viewpoint of the two main research questions (1) what are the applications of Al for COVID-19 pandemic and (2) how well do Al techniques perform for COVID-19 pandemic? The objective of this SLR is to explore, examine, and summarize the Al techniques employed by researchers for containment COVID-19 pandemic. The search strategy began by determining the search period. Research published between December 2019 and May 2020 has been retrieved. The starting date (January 2010) was selected because the first positive case recorded for COVID-19 was identified on December 2019 in Wuhan, China. While, the end date (December 2019) was selected because the SLR was conducted during this period. Next, research sources were identified from which relevant Al applications for COVID-19 research will be retrieved. Seven search resources (e.g., ScienceDirect-Elsevier, IEEEXplore, PubMed, Embase and Google Scholar) were considered to include broadly related papers published and preprint during period. Subsequently, search keywords 
and phrases have been identified. Table 2 shows the dimensions and keywords, alternative keywords and keywords synonyms of Al for COVID-19 pandemic that applied to conduct the automatic search.

\begin{tabular}{|c|c|c|}
\hline & Dimensions & Keywords, alternative keywords and keywords synonyms \\
\hline \multirow{4}{*}{ AND } & Artificial intelligent & $\begin{array}{l}\text { Machine Learning OR Neural Network OR Expert Systems OR Fuzzy } \\
\text { Logic OR Case-Based Reasoning OR Deep Learning OR Meta Heuristic } \\
\text { Algorithms OR Support Vector Machine OR k-Nearest Neighbor OR } \\
\text { Naive Bayes Classifier Optimization OR Algorithms. }\end{array}$ \\
\hline & Purpose & $\begin{array}{l}\text { Prediction OR Forecasting OR Diagnosis OR Detection OR Tracking OR } \\
\text { Distinguishes OR Managing OR Classification. }\end{array}$ \\
\hline & COVID-19 & $\begin{array}{l}\text { Coronavirus OR SARS-CoV-2 OR Pandemic OR Epidemic OR Outbreak } \\
\text { OR Disease OR Infection. }\end{array}$ \\
\hline & Healthcare & Drugs OR Medicine OR Vaccines OR X-ray OR Computed Tomography \\
\hline
\end{tabular}

The number of papers retrieved from the initial automatic search is 3690 papers. By reviewing the retrieved papers titles and abstracts, 3460 papers were excluded because they were beyond the scope of this SLR. The remaining 230 research papers were filtered using inclusion and exclusion criteria. Therefore, papers that matched the following inclusion criteria were included:

- Papers related to the COVID-19

- Papers presenting Al

It is worth noting that all published and pre-prints papers are included. Although pre-prints papers still need to be peer-reviewed and evaluated for approval by the scientific community, it was included in the study because (1) to cover the largest possible number of Al techniques for COVID- 19 Pandemic (2) they start getting citations from other researchers. This means that many of the current research related to the applications of Al techniques for COVID-19 pandemic started relying on these pre-prints researches which could affect the building of the body of knowledge in this research area. So, we found it necessary to include, review and investigate them.

While, papers that matched at least one of the following exclusion criteria were excluded:

- Papers not focusing on the Al for COVID-19

- Papers providing only recommendations, guidelines, or principles for Al for COVID-19 pandemic

- Papers not written in

To answer the main research questions, 73 research papers were indexed in Appendix $A$ and each paper was read in-depth. As presented in Table 3, data extraction form that consist of 12 dimensions (e.g., Application of Al (AoAl), ID, Source, Purpose, Al-Methods, Validation Approach, Limitations, Data set used for, Name of dataset, Size of data set, Country, Source of dataset) is created to (1) facilitates the process of data extraction (2) organize and record full details of the papers under review and (3) specify how each research question was addressed. 


\subsection{Study Distribution}

Figure 1. present the distribution of the selected papers from December 2019 to May 2020 from different electronic resources. By applying search strategy and paper filtering process, 73 papers were selected for the review study. In detail, the highest number of papers (e.g., 25 papers) were retrieved from medRixv. Following by 23 papers from arXive, 3 papers were retrieved from each of Biorxiv and SSRN. The remaining papers (e.g., 19 papers) were retrieved from different electronic resources as shown in Figure 1.

\section{Applications Of Ai Against Covid-19}

The review result revealed that Al techniques have been applied to various research areas related to COVID-19. For instance, Al has been employed to COVID-19 outbreak prediction, predicting criticality of COVID-19 cases, distinguishes COVID-19 from chest X-ray, distinguishes COVID-19 from chest CT images, distinguishes COVID-19 from clinical test, distinguishes COVID-19 from genomic signatures, distinguishes COVID-19 from sound signal, detecting and tracking COVID-19 cases, developing COVID-19 drugs and vaccines, and managing healthcare resources-COVID-19. A brief summary of the main applications of $\mathrm{Al}$ is shown in Table 3 and discussed in the following subsequent sections.

\subsection{Predicting COVID-19 Cases}

\subsubsection{COVID-19 Outbreak Prediction}

A serious challenge in the sustainable improvement manner became investigated the usage of the category of confirmed cases of COVID-19 as one of the epidemic diseases. For this reason, binary category modeling was utilized by the institution method of records coping with (GMDH) kind of neural community as one of the synthetic intelligence strategies [S1]. For this motive, the Hubei province in China changed into selected as a case look at to construct the proposed model, and a few critical factors, namely maximum, minimum, and common day by day temperature, the density of a town, relative humidity, and wind velocity, have been taken into consideration as the input dataset, and the number of confirmed instances become decided on as the output dataset for 30 days. The proposed binary class version gives higher performance capability in predicting the confirmed instances. Similarly, regression evaluation has been accomplished and the trend of confirmed cases compared with the fluctuations of everyday weather parameters (wind, humidity, and average temperature). The outcomes verified that the relative humidity and most days by day temperature had the best effect on the showed instances.

In the current study, a new forecasting version is supplied to estimate and forecast the quantity of showing cases of COVID-19 in the following ten days based totally on the previously confirmed cases recorded in China. The proposed version is a progressed adaptive Neuro-fuzzy inference machine (ANFIS), the use of a better flower pollination set of rules (FPA) with the aid of using the salp swarm set 
of rules (SSA). In well-known, SSA is employed to enhance FPA to avoid its drawbacks (e.g.,getting trapped at the neighborhood optima) [S2]. The original idea of the proposed model, referred to as FPASSA-ANFIS, is to enhance the performance of ANFIS by figuring out the parameters of ANFIS the use of FPASSA. The FPASSA-ANFIS version has evaluated the use of the sector health agency (WHO) official statistics of the outbreak of the COVID-19 to forecast the confirmed instances of the following ten days. Extra so, the FPASSA-ANFIS model is compared to numerous existing models, and it confirmed higher overall performance in phrases of suggesting Absolute percent blunders (MAPE), Root mean Relative Squared mistakes (RMSRE), Root suggests Squared Relative errors (RMSRE), coefficient of dedication (R2), and computing time. Furthermore, the authors examined the proposed model the use of different datasets of weekly influenza showed instances in two nations, specifically the United States and China. The outcomes also confirmed proper performances.

A strong records-pushed machine learning evaluation is presented for the COVID-19 pandemic from its early infection dynamics; specifically, contamination counts over the years [S3]. The intention is to extract actionable public fitness insights. These insights include the infectious force, the charge of mild contamination becoming serious, estimates for asymptomatic infections, and predictions of the latest infections over time. They recognition on U.S.A. records starting from the first showed infection on January 20,2020. Their strategies monitor large asymptomatic (hidden) infection, a lag of about ten days, and we quantitatively affirm that the infectious force is powerful, with approximately a $0.14 \%$ transition from slight to critical infection. Their techniques are efficient, robust, and trendy, being agnostic to the precise virus and applicable to one of a kind populations or cohorts.

COVID-19 is spreading all across the globe. Up till March 23, 2020, the showed cases in 173 nations and areas of the globe had passed 354,000 , and extra than 15,300 deaths had resulted. The confirmed cases outdoor of China had also reached over 81,000, with over 3, two hundred deaths. On this look at, a Convolutional Neural network (CNN) changed into proposed to analyze and predict the quantity of showed instances. Several towns with the most confirmed cases in China have been the point of interest of this take a look at, and a COVID-19 forecasting version, primarily based on the CNN deep neural community technique, become proposed [4]. To compare the general efficacies of different algorithms, the signs of mean absolute mistakes and root suggest rectangular errors become applied in the test of this take a look at. The test outcomes indicated that as compared with other in-depth gaining knowledge of methods, the CNN model proposed on this examination has the best prediction efficacy. The feasibility and practicality of the version in predicting the cumulative wide variety of COVID-19 showed instances.

The current international outbreak of COVID-19 sickness is affecting many countries globally. Iran is one of the pinnacle 10 maximum affected countries. Search engines provide useful data from populations, and these statistics might be useful to research epidemics. Utilizing records mining strategies on electronic assets' information would possibly offer a higher insight into the COVID-19 outbreak to manipulate the health disaster in every case and worldwide. This takes a look at aimed to expect the incidence of COVID-19 in Iran. Methods: records have been received from the Google traits website. Linear regression and lengthy short-time period memory (LSTM) models were used to estimate the wide variety 
of active COVID-19 cases. All models had been evaluated using 10- fold go-validation, and root imply rectangular blunders (RMSE) become used because of the overall performance metric. The linear regression model anticipated the incidence with an RMSE of seven.562 (SD 6.492). The most effective factors besides previous day occurrence included the hunt frequency of hand washing, hand sanitizer, and antiseptic topics. The RMSE of the LSTM version changed into 27.187. Data mining algorithms may be employed to expect traits of outbreaks. This prediction might help policymakers and fitness care managers to plan and allocate health care resources accordingly [S5].

Even though COVID-19 has been nicely controlled in China, it is far rapidly spreading outside the US. It can have catastrophic results globally without the implementation of vital mitigation measures. Because the COVID-19 outbreak has made complete and profound impacts on the world, an accurate prediction of its epidemic fashion is sizable [S10]. Even though much research has predicted the COVID-19 epidemic trend, maximum have used early-stage records and centered on Chinese language cases. They first built fashions to predict each day's number of cumulative confirmed instances (CCCs), new instances (NCs), and loss of life instances (DCs) of COVID-19 in China based on records from January 20, 2020, to March 1,2020 . Based on these models, they constructed models to predict the epidemic trend the world over (outdoor China) [S6]. Additionally, they built models to be expecting the epidemic fashion in Italy, Spain, Germany, France, UK, and US. Wherein COVID-19 is unexpectedly spreading. The COVID-19 outbreak may have peaked on February 22, 2020, in China and will top on May also 22, 2020, internationally. It will be below manipulated in early April 2020 in China and cease-August 2020 across the world. The whole variety of COVID-19 cases will reach around 89,000 in China and six, 126,000 across the world in the course of the epidemic. Round four, 000, and 290,000 people will die of COVID-19 in China and the world over, respectively. The COVID-19 outbreak can have peaked recently in Italy and could peak in Spain, Germany, France, the UK, and the US inside weeks. Interpretation: The COVID-19 outbreak is controllable within the foreseeable destiny if comprehensive and stringent manipulate measures are taken.

This 21 st century is remarkable for witnessing so many upheavals at social, cultural, economic, and political levels in India. These changes have affected the lives of every Indian drastically [. Nowadays, a new challenge is in front of us all, which has not only made our present miserable with its dreadful consequences, but it will also not leave its painful clutches to spare our future life. The name of this threat is COVID-19, a highly contagious virus whose ruthless grip has left humanity to breathe in fear and danger. The coming week holds the key to whether India will stay on course to enter the 'steady phase' of the COVID-19 pandemic by April 16. According to data available with the government, which has been discussed at the highest levels, India is currently in the 'acceleration phase.' Keeping this in mind, we have tried to use machine learning to analyze the current situation created by COVID-19 and what may be its impact in the coming days [S7].

From late December 2019, the novel COVID-19 started to spread inside the mainland of China. For predicting the fashion of the COVID-19 unfolds, numerous time delay dynamic structures (TDD) are proposed. In this paper, we set up a different fractional time put off dynamic device (FTDD) to describe the neighborhood outbreak of COVID-19. The fractional by-product is introduced to account for the sub- 
discussion system of the confirmed and cured peoples' boom. Based totally on the general public fitness records through the government, we advise a robust reconstruction to set of rules of the coefficients. The reconstructed coefficients are used to predict the trend of the COVID-19. The numerical outcomes are in precise agreement with the general public statistics [S8].

Unsupervised system mastering algorithms (okay-method) were used to define records-pushed clusters of countries; the set of rules became informed with the aid of ailment prevalence estimates, metrics of air pollution, socio-monetary fame and health gadget insurance. The usage of the only-manner ANOVA test, we as compared the clusters in phrases of the number of showing COVID-19 cases, a wide variety of deaths, the case fatality rate, and order in which suggested the first case. The version of outlining the clusters changed into developed with 155 international locations [S9]. The model with the three most crucial factor evaluation parameters and five or six clusters showed the exceptional ability to organize nations in applicable units. There was definite proof that the version with five or six clusters may want to stratify international locations consistent with the wide variety of confirmed COVID-19 cases ( $p<$ zero.001). However, the version could not stratify countries in phrases of quantity of deaths or case fatality price. A secure statistics-driven method the use of to be had international facts before the COVID19 pandemic regarded able to classify nations in phrases of the wide variety of confirmed COVID-19 instances. The model turned into now not able to stratify international locations based on COVID-19 mortality records.

Machine learning has been employed for a set of numerical models dependent on different factors and investigations are dependent upon potential inclination. In [S11], the authors proposed a basic model that could be useful to foresee the spread of COVID-19. While, in [S12], the authors introduced a novel approach that integrated both of digital traces and mechanistic models using machine learning to estimate the COVID-19 epidemic. The results of the work could be used as input for (1) News media by Media Cloud. (2) COVID-19 linked internet search action. (3) Formal health determinations from Chinese Center Disease for Control and Prevention (China CDC). The goal of employing machine learning to of geo-spatial synchronicities of COVID-19 action in Chinese provinces. The results showed that the proposed approach outperformed a set of the baseline approaches.

Study proposed by [S13], machine learning tools and SIR model have been utilized to analyze the COVID19 epidemic. They estimated the major point of the epidemic parameters. After that, determined the area around the point to predict the infection and find the feasible ending time. Based on their hopeful estimation, the epidemic will disappear soon in some countries. While, Hu et al. [S14] proposed methods inspired by Al to forecast the transmission dynamics of COVID-19 in China. The proposed methods aim to estimate the lengths, size, and ending time of COVID-19 across China. The authors utilized the latent elements in the clustering and encoding algorithms. The results showed that the proposed method achieved high accurately forecast the transmission dynamics of the Covid-19 which aid to provide suitable plans and policymaking. 
Punn et al. [S15] employed machine learning and deep learning algorithms to analyze the COVID-19 epidemic. The work aimed to study all exponential behavior every day, which aid to increase the predicts ability of the COVID-19 epidemic in the future using real-time information. Thus, reducing the number of susceptible individuals from infected individuals. In [S16], the authors integrated a multi-layer feedforward neural network (MFNN) and improved interior search algorithms (ISA) based on chaotic learning (CL) that called ISACL-MFNN, for COVID-19 forecasting. CL has been used to improve the performance of the ISA, as well as enhance its exploitation ability. Also, MFNN has been utilized to deal with the parameter setting which aid to get a high-accuracy level in forecasted results. The experimental results showed that the proposed method achieved high performance in a forecasting task comparing with the other algorithms in the literature. While in [S17], the authors introduced a comparative analysis of machine learning to predict the COVID-19 outbreak. Both studies proved the efficiency of the machine learning to deal with the outbreak.

Distante et al. [S18] benefited from the mechanism by which the Chinese dealt with the COVID- 19 crisis to employ it in the Italy. The authors connected the events that occurred in both countries, then used the neural networks to the forecast epidemic curve of the various Italian areas and use the susceptibleexposed-infected removed (SEIR) compartment model to predict the spreading and peaks. Based on that, the authors match the similarity of the Italian results with the Chinese and how the last deal with it. In other words, utilizing training neural networks on the Chinese experience and employ it to forecast Italian pervasion of COVID-19 proved its efficiency. In [S19], the top fifteen countries of COVID-19 Pandemic have been introduced for forecasting the dynamics using machine learning and ARIMA Model. The results of the analysis predicted were unexpected and frightening, where all selected countries will experience chaos. Thus, governments should be more stringent to grasp the power exponential growth.

\subsubsection{Predicting criticality of COVID-19 cases}

The random forest classification algorithm is proposed in [S20], in order to classify the risk factors associated with the death of COVID-19 infected persons. Around 1085 cases of clinical data of COVID-19 have been collected between January and February 2020 from the online community of Data scientists "Kaggle". This dataset goes through a preprocessing phase to clean missing data, and 430 cases were nominated for the examination. Results show that the area under the ROC curve is 0.97 which indicates good accuracy of classification, also patients more than 62 years old are at greater risk.

A database of blood samples from 404 infected patients in the region of Wuhan, China were collected in [S21] to identify crucial predictive biomarkers of disease severity. A supervised XGBoost classifier using recursive tree-based decision system was applied, three biomarkers that predict the survival of individual patients were selected with more than $90 \%$ accuracy: lactic dehydrogenase (LDH), lymphocyte and highsensitivity C-reactive protein (hs-CRP). High levels of LDH were shown to have a huge effect on most of the cases that require immediate intensive care. In addition, results show that Lymphopenia is a common feature in patients with COVID-19 and might be a critical factor associated with disease severity and mortality. 
In [S22] a statistical model was built to determine prognostic risks of COVID-19 patients. A set of 669 COVID-19 pneumonia patients confirmed were collected for statistical analyses and modeling. The collected data include Real-time nucleic acid-PCR, radiologic CT, and hematologic laboratories. These data were used to build the Cox regression model, where the patients are clustered into two groups, death, and survival group. Two different tests were used based on attribute types. T-tests applied on continuous variables, while for categorical A Chi-square test was applied. The model results indicate sensitivity of 0.8172 and specificity of 0.7858 . Results indicate that the COVID-19 has a high risk of older people with chronic diseases where they are more likely to die

An implementation of five different machine learning algorithm have been proposed in [S23], in order to predict the risk of positive diagnosis for COVID-19. A data set of 235 adult were collected from a hospital in Brazil, which includes 10 positive cases for COVID-19. In this research, they applied neural networks, random forests, gradient boosting trees, logistic regression and support vector machines. That Results show better accuracy with support vector machine, as the area under the curve was 0.85 ; Sensitivity: 0.68 and Specificity. Thus, it can be easier to suggest that targeted decision for receiving COVID-19 tests in areas with a shortage of supply.

The first implementation for the deep learning algorithm was presented in [S24]. The main objective was to decide if it is practical to apply deep learning to evaluate how much prediction results are close to original data related to Confirmed-Negative Released-Death cases of Covid-19. A dataset of Kaggle for patients in South Korea was selected and a framework of long short-term memory (LSTM) and Gated Recurrent Unit (GRU) is used for the dataset training. The method shows a very high prediction rate and fast identification of COVID-19.

A method for predicting the mortality risk of patients with COVID-19 was presented in [S25]. Various machine-learning algorithms (e.g Support Vector Machine (SVM), Artificial Neural Networks, Random Forest, Decision Tree, Logistic Regression, and K-Nearest Neighbor (KNN)) were applied to compare the best accuracy. A data set of 117,000 patients world-wide with laboratory-confirmed COVID-19 were used. Results show better performance for Rando Forest algorithm in terms of AUC and accuracy. This proposed method can be very helpful in different medical facilities to decide patients who needs high attention before others when the medical system is overwhelmed.

In [S26] the XGBoost machine learning has been applied to predict the mortality risk in patients before they transmit to critically ill. A data of 2,799 patients admitted in Tongji Hospital from January 10th to February 18th, 2020 have been gathered. Results of proposed algorithm show that the mean age of patients was 58.83 years old with $58.7 \%$ of males, followed by cough (13.9\%), fatigue (3.7\%), and dyspnea (2.1\%). The proposed model recognized three key clinical features, e.g.,lactic dehydrogenase (LDH), lymphocyte and High-sensitivity C-reactive protein (hs-CRP), out of the all 300 features in the dataset to be more accurate in predicting mortality risk. The will help in the recognition of critical cases from severe cases. 
Also the same XGBoost machine learning has been proposed in [S27] to predict the survival for patients with severe COVID-19. 375 cases of clinical data collected from Tongji Hospital, Wuhan was used for training the model, while other new 29 patient cases were used for the evaluation process. The proposed model achieved accuracy more the $90 \%$ in predicting survival patients. But this model concentrates on only three main features e.g.,lactic dehydrogenase (LDH), lymphocyte and High-sensitivity C-reactive protein (hsCRP), in which the model can be applied on other different features to get higher accuracy rate.

In [S28] a new Patient Information Based Algorithm (PIBA) is presented. The algorithm is applied for estimating the death rate of a disease in real-time. A large data set collected from three public hospitals in China, multi pre-processing steps applied to the collected data before implementing the PIBA. The accurate death rate of COVID-19 in China ranges from $0.75 \%$ to $3 \%$ and results showed that the real death numbers in China have fallen into the predicted ranges. This indicates that PIBA can predict the death rate of a new infectious disease in real-time also.

\subsection{Diagnosing COVID-19 Cases}

\subsubsection{Distinguishes COVID-19 From Chest X-ray}

The COVID-19 pandemic is placing the best healthcare systems around the world under tremendous pressure. Detecting this virus in the early stage will help the healthcare systems, doctors, and governments to relieve the pressure of the COVID-19. The chest X-ray and CT images are significant methods to diagnose this disease. Artificial intelligence (Al) algorithms, used widely to diagnose different pneumonia. Several frameworks proposed to detect the COVID-19 based on Al algorithms.

In [S29] a deep convolutional neural network was proposed to classify chest X-rays of patients who have tested positive for COVID-19. The data have been collected from two sources, the first part of GitHub COVID-19 image data collection repository and the second part of RSNA Pneumonia Detection Challenge dataset available on Kaggle. Both datasets include CXRs and CT scans from COVID-19 cases. An Interpretable Model-Agnostic Explanations (i.e. LIME) has been applied on the datasets after cleaning. Results indicate high accuracy for predicting positive COVID-19 cases.

In a term of distinguishing between the COVID-19 patients and healthy individual patient. Kumar, Rahul, et al (2020) [S30]. Propose a classification method based on the ResNet152 model to find the deep features of COVID-19 and another type of pneumonia using X-ray images. To balance the imbalanced data of COVID-19 and other diseases, the authors suggest using the synthetic minority oversampling technique (SMOTE) model. The result 0:977 of accuracy by XGBoost classifiers, and 0.973 using Random forest. In study proposed by Castiglioni et al., 2020 [S31], to diagnose COVID-19 using X-ray images, the TRACE4 framework employs for image analysis as an Al approach. Then, ResNet-50 adapted to use as a classifier [S38]. Hassanien, Aboul Ella, et al. [S32] uses the X-ray images for diagnosis the COVID-19 by using the median filter to improve the contract of the images. The Ostu objective approach applied to the multiimage segmentation threshold. Then, the SVM algorithm employs for classifying COVID-19 patients 
[S37]. The class decomposition approach used to classify variations in the X-ray image dataset by examining its class boundaries [S33]. Pereira, Rodolfo M., et al. [S34] propose to use various texture descriptors to observe the visual features of the chest X-ray, for an instant Local Binary Pattern (LBP), Elongated Quinary Patterns (EQP), Local Phase Quantization (LPQ), etc. Then use the Early fusion approach, which is used to group several features as one group of features. Late Fusion approach will be used after resampling the dataset to combine the output. Alqudah, Ali Mohammad, et al. presented an approach based on CNN to classify the visual features of X-ray images, SVM, and Random Forest [S35]. A deep convolutional neural network to detect the COVID-19 proposed by Wang \& Wong, 2020 [S36], and the GSInquire approach used to ensure the result of detection based on relevant information, not incorrect information. A CNN architecture and gravitational search algorithm (GSA) for optimization, to choose the best value for CNN architecture [S37]. Studies introduced by [S39] and [S40] propose Trained CNN for COVID-10 detection in X-rays. [S41] proposes an approach based on Capsule Network method called COVID-CAPS, the proposed framework diagnosis the COVID-19 for X-ray and CT.

\subsubsection{Distinguishes COVID-19 From CT Images}

Li et al., (2020) [S42] proposed a method named COVID-19 observation neural network (COVNet), this approach uses the ResNet50 to extract 2D and 3D CTimages features. Then, the extracted feature combines by the max-pooling operation. Finally, the softmax activation approach used to generate the probability score of COVID-19 or usual pneumonia. In study [S43], to improve the detection process in CT system, several feature extraction approaches used like Local Directional Pattern (LDP), Grey-Level Size Zone Matrix (GLSZM), Grey Level Co-occurrence Matrix (GLCM), Discrete Wavelet Transform (DWT), and Grey Level Run Length Matrix (GLRLM). For feature extraction, the SVM algorithm proposed to use in this study. The result demonstrates that accuracy reaches $99.68 \%$ by the GLSZM approach. Hu et al., (2020) [S44] proposes weakly supervised deep learning approach to detect the COVID-19 from CT images.

In terms of enhancing the performance of the computerized tomography (CT) scan and perform a realtime diagnosis of COVID-19, [S45] proposes a Joint Classification and Segmentation (JCS) method. The Res2Net network approach used to classify the patients if they have the disease or no based-on CT images. The activation mapping approach used to improve accountable transparency. Then the global average pooling (GAP) employ to reduce the size of the feature. For segmentation, the proposed method uses encoder and decoder based on different algorithms such as VGG and Attentive Feature Fusion (AFF), Squeeze-Excitation (SE) to enhance the segmentation process. The result showed $95 \%$ accuracy in the classification and $78.3 \%$ in segmentation. Similar to the previous study [S46] propose a multitask deep learning approach to identify COVID-19 jointly.

Based totally on COVID-19 radiographical changes in CT photos, they hypothesized that synthetic Intelligence's deep learning methods might be capable of extract COVID-19's precise graphical capabilities and provide a clinical analysis in advance of the pathogenic take a look at, as a result saving essential time for ailment control. To check this opportunity, they amassed 453 CT snapshots of pathogenconfirmed COVID-19 cases together with formerly recognized with common viral pneumonia [S47]. Two 
hundred seventeen snapshots were used as the education setting, and the inception migration-studying model turned into used to set up the algorithm. The internal validation carried out a complete accuracy of 82.nine $\%$ with a specificity of 80. five $\%$ and a sensitivity of eighty-four $\%$. The external trying out dataset showed a total accuracy of seventy- three $.1 \%$ with a specificity of sixty-seven $\%$ and sensitivity of seventy-four \%. These effects imply the superb cost of the use of the in-depth, gaining knowledge of the method to extract radiological graphical functions for COVID-19 diagnosis.

In this paper, they use artificial intelligence (Al) algorithms to combine chest CT findings with medical signs, exposure records, and laboratory testing to more accurately and rapidly diagnose SARS-CoV-2 (+) sufferers [S48]. They protected 905 RT-PCR showed patients. 419 (forty six.2\%) tested effective for SARSCoV-2 by using laboratory-showed real-time RT-PCR assay and subsequent-era sequencing, at the same time as 486 sufferers (53.eight\%) examined bad (confirmed with the aid of at the least two additional bad RT-PCR checks and scientific commentary). The proposed Al device did an AUC of 0.ninety two and performed similarly nicely in sensitivity as compared to a senior thoracic radiologist on a trying out a set of 279 instances. The Al machine also stepped forward the detection of RT-PCR high-quality SARS-CoV-2 sufferers. They supplied with everyday CTs, successfully figuring out $17 / 25(68 \%)$ patients. In contrast, all 25 RT-PCR SARS- CoV-2-advantageous CT-ordinary patients had been categorized as SARS-CoV-2 poor through radiologists.

In this paper [S49], a unique method changed into proposed as fusing and rating in-depth features to stumble on COVID-19 in the first section. 16x16 (Subset-1) and 32×32 (Subset-2) patches were obtained from 150 CT pictures to generate sub-datasets. Within the scope of the proposed technique, 3000 patch images had been labeled as CoVID-19 and No locating for use in the schooling and testing segment. A unique fusion and rating approach had been carried out if you want to boom the performance of the proposed method. Then, the processed information became labeled with an aid Vector system (SVM). According to other pre-skilled Convolutional Neural community (CNN) fashions used in switch studying, the proposed technique shows high overall performance on Subset-2 with $98.27 \%$ accuracy, $98 \%$ sensitivity, $97.60 \%$ specificity, $97.63 \%$ precision, $98.28 \%$ F1-rating and $96.54 \%$ Matthews Correlation Coefficient (MCC) metrics.

To assess imaging features and completed quantitative evaluation for moderate novel COVID-19 instances equipped for discharge [S50]. CT pix of a hundred twenty-five sufferers (16-sixty-seven years, sixty-three men) improving from COVID-19 were tested. They described the double-poor duration (DNP) because of the period between the sampling days of consecutive bad RT-PCR and three days after that. Lesion demonstrations and distributions on CT in DNP (CTDN) were evaluated through radiologists and artificial intelligence (Al) software. Foremost lesion transformations and the involvement range for sufferers with complies with-up CT were analyzed. Twenty (16.0\%) sufferers exhibited normal CTDN; extraordinary CTDN for one hundred and five indicated ground-glass opacity (GGO) (ninety-nine/one hundred twenty-five, 79.2\%) and fibrosis (56/one hundred twenty-five, $44.8 \%$ ) as the maximum frequent CT findings. Bilateral-lung involvement with blended or random distribution turned into most commonplaces for GGO on CTDN. Fibrous lesions regularly affected each lung, tending to distribute on 
the sub pleural. Follow-up CT confirmed lesion improvement manifesting as GGO thinning (40/40, one hundred\%), fibrosis discount (17/26, 65. four\%), and consolidation fading (Sept. 11, eighty-one. eight\%), without or with variety reduction. Al evaluation showed the highest proportions for right lower lobe involvement (extent, $12.01 \pm 35.87 \mathrm{~cm} 3$; percentage; $1.45 \pm$ four.58\%) and CT-fee ranging -570 to $-470 \mathrm{HU}$ (quantity, 2.ninety three $\pm 7.04 \mathrm{~cm} 3$; percentage, five. $28 \pm 6$. forty seven\%). Among instances with follow-up $\mathrm{CT}$, the maximum of lung lobes and CT-value degrees displayed a significant reduction after DNp. The principle CT imaging manifestations have been GGO and fibrosis in DNp, which weakened with or without quantity reduction. Al evaluation results have been constant with imaging functions and adjustments, likely serving as a goal indicator for disorder monitoring and discharge.

This paper reports on the improvement and performance of device studying schemes for the analysis of Chest CT test photos of COVID-19 patients and demonstrates massive achievement incorrectly and robotically trying out for COVID-19 contamination [S51]. In particular, a new frequency domain set of rules, to be called the FFT-Gabor scheme, can be shown to be expecting in nearly real-time the state of the patient with a mean accuracy of $95.37 \%$, sensitivity ninety-five. Ninety-nine $\%$ and specificity $94 \%$, Seventy-six \%. The FFT-Gabor scheme is satisfactorily informative in that clinicians can visually examine the FFT-Gabor function to aid their final diagnostic.

\subsubsection{Distinguishes COVID-19 From Clinical Test}

In [S52], novel clustering tools of Al-assisted diagnosis helps the system to suspect COVID-19 pneumonia. The authors based on the features from routine laboratory tests, clinical symptoms, and clinical data on admission. The proposed strategy achieved good results in various tests, such as accuracy of 0.400 and 0.500 , the specificity of 0.727 and 0.778 , and recall of 1.000 and 1.000 . Thus, using the proposed strategy allow to early detect just on admission in fever clinics.

Peng et al. [S53] utilized Al to analyze the COVID-19 general diagnosis index for improving the accuracy of the diagnosis in the clinical area. The authors employed four various types of Al techniques to check the index of COVID-19 diagnosis. The works aim to reduce the features of selection techniques. The results illustrated that the Al techniques achieved 18 indexes related to significant COVID-19 diagnosis.

In [S55], the authors proposed a new method based on blood test results and machine learning model for identifying the COVID-19 with high accuracy and rapid. Although 19 various cases suspected patients have been chosen, the results of the proposed method were robust and accurate (e.g.,the accuracy of 0.9795 for the cross-validation set and 0.9697 for the test set). The proposed method proved its efficiency to be a reliable technology and has the ability to reduce the burden of global public health.

Zhang et al. [S56] used urine and blood tests with machine learning to detect the severity of COVID-2019 on patients. The authors employed the support vector machine (SVM) on 75 cases suffer severely and 62 cases suffer mild symptoms. The results illustrated that the SVM proved its efficiency by detecting 32 features (e.g.,overall accuracy 0.8148). 
In [S57], Al and simple blood exams have been used to propose a novel high specificity COVID- 19 screening. The authors employed a development version of machine learning to get the inputs from widely obtainable blood exams. Depends on the first classification, the positive cases (having SARS-CoV2) can be indicated for more highly sensitive examine, such as specific antibodies or CT scans.

\subsubsection{Distinguishes COVID-19 From Genomic Signatures}

To overcome the diagnostic challenges related to the current SARS-CoV-2 outbreak and the need for a rapid and fast diagnostic method, a system called ADAPT is proposed using algorithms and machine learning models for rapidly designing nucleic acid detection assays [S58]. The proposed algorithm to detect 67 viral species and subspecies, including SARS-CoV-2, phylogenetically related viruses, and viruses with similar clinical presentations. The system has been evaluated based on the sensitivity of the SARS-CoV-2 assay against clinical isolates and patient samples, and specificity at both the species and subspecies levels against highly related viruses. It was suggested to include a mixture of synthetic targets reflecting different viral sequences, and patient samples or viral seed stocks

In [S59] a method for identifying an intrinsic COVID-19 genomic signature, using a machine learningbased alignment-free approach for classification of the whole COVID-19 genomes are implemented. A combination of a supervised machine-learning algorithm and the digital signal process is applied for genome analyses. A decision tree was implemented as the machine-learning component. For validating the results, a Spearman's rank correlation coefficient analysis is done over 5000 different viral genomic sequences. Results proved the hypotheses of the bat origin of COVID-19. In addition, the applied alignment-free whole-genome classification approach can be very helpful in real-time taxonomic classification.

\subsubsection{Distinguishes COVID-19 From Sound Signal}

An artificial Intelligence transfer learning algorithm is proposed for diagnostic tests of COVID- 19 based on cough phone recordings from multiple patients [S60]. As deep learning can be very useful for analysis of voice data, thus an unsupervised transfer learning was applied. This method has been tested by pretraining DenseNet201 and ResNet50 architectures with samples from the speech dataset. The algorithm was tested on a dataset from 150 patients and 3000 contacts as part of the clinical trial motioned, where each sample is 12 seconds. The results were validated in a clinical trial and three other venues in Mexico, Spain and the USA1.

\subsection{Detecting \& Tracking COVID-19 Cases}

In response to the recent outbreak of COVID-19, study in [S61] proposed a new model of active monitoring for COVID-19 by artificial intelligence. Both past and current incidents of outbreaks of infectious disease have shown that the unavailability of successful methods of screening the whole population and attempts is actually stopping the pandemic. Furthermore, social distancing and home quarantine at this 
stage are only measures to avoid the spread of COVID-19 disease. The presented project aimed to implement a reliable approach of using speech-recognition technologies via a mobile app to identify the cough sounds of suspicious people who were previously healthy, suffer from respiratory illness, and actively monitor their symptom progress in real time. The mobile application regularly provides feedback to users of the app and advise them to take medication and the appropriate precautions to avoid spreading the disease. The app also notifies sick users to nearby health care facilities, or vice-versa, if their symptoms worsen. A feedback is sent to an app-center, informing the number of new and old sick users in a given area. With the data recorded at the app-center, it will also be able to track disease spread patterns, classify regions into three categories that are mild, moderate and severe based on the number of sick users and deploy the resources appropriately to stop the transmission thereby preventing excessive use of both human and medical resources to avoid the infection in redundant areas.

Study presented by [S62] proposed a novel bioinspired metaheuristic, which simulated how the COVID-19 spread and afflicts healthy people. The COVID-19 infects patients at known levels from an initial person (the patient zero), thus creating new populations of infected people. The individual can either die or become infected, and then be returned to the recovered people. In the model, appropriate terms such as probability of re-infection, super-spreading rate or traveling rate are used to simulate the COVID-19 behavior as accurately as possible. Compared with other related approaches, the COVID-19 Optimization Algorithm has two significant advantages. First, according to the disease statistics, the input parameters are already set, preventing researchers from initializing them with arbitrary values. Secondly, the method has the potential to end after many iterations, without this value being set either. Infected population briefly increases at an exponential rate, but after several iterations the number of infected people begins to decrease in subsequent iterations when examining social isolation measures and the large number of recovered and dead people. In addition, a parallel multi-virus model is suggested, in which many strains of COVID-19 evolve over time and exploit large search spaces in less iterations. At last, in order to find suitable hyperparameters during the training process, the metaheuristic was combined with deep learning methods. The problem of electricity load time series forecasting was tackled as application scenario, showed very remarkable results.

A study conducted by [S63] recommends utilizing machine learning algorithms to enhance probable COVID-19 case identification quickly by using a web survey based on the mobile phone. This may decrease the spread of susceptible populations as well. While, study proposed by [S64] aims to explain and extrapolate from publicly accessible data using a mixed epidemiological equation of first principles and a data-driven model of the neural network. Taking advantage on the neural network enhanced model, it's concentrated in this study on four locations: Wuhan, Italy, South Korea and the United States of America, and compare the role of quarantine and isolation measures in each of these places in managing the virus' successful Rt reproduction number. Findings indicate unequivocally that the countries where rapid government initiatives and stringent quarantine and isolation measures for public health have been introduced have been effective in halting the spread of infection and preventing it from exponentially exploding. 
The latest worldwide epidemic of the COVID-19 introduced the scientific community to new challenges [S65]. Methods powered by artificial intelligence (AI) can be valuable for predicting the parameters, risks and consequences of such an epidemic. Such predictions can be of assistance in controlling and preventing the spread of such diseases. The major challenges of using Al are the small quantity of data and the uncertain nature of the data. Thus, to predict a country's risk category, the authors suggests a shallow short-term memory (LSTM) based neural network. The authors employed a Bayesian optimization model to optimize country-specific networks, and design them automatically. For the prediction it mixed trend data and weather data together. Results showed that the presented pipeline outperforms data for 170 countries against state-of-the-art methods and could be a valuable tool for categorizing these risks. The method could also be employed to predict such an epidemic's long-term outbreak so it can take preventive measures earlier.

Italy actually constitutes the epicenter of the pandemic of the novel COVID-19 disease (COVID- 19), having exceeded the number of deaths in China. The illness is spreading across Lombardy, which has been in lockdown since March 8. The isolation steps taken in Lombardy is extenuated to the whole world as of the same day. In this study, the authors suggested estimates for: (a) the DAY-ZERO of the epidemic in Lombardy, Italy; (b) the real number of cases of exposure/infection in the total population; (c) the simple reproductive number (R0); (d) the 'successful' regular transmission of disease; and, most significantly, (e) a prediction of the epidemic fades, based on the COVID-19 Community Mobility Reports published by Google on 2 March [S66]. To tackle the ambiguity in the number of real uncovered / diseased cases in the overall population, the authors in this study tackle a compartmental Susceptible/ Exposed / Infectious / Recovered / Dead (SEIRD) model with two compartments of infectious individuals: one modeling the overall population diseases and another modeling the reported cases. Specifically, simulations showed that the COVID-19 outbreak is predicted to fade out in Lombardy by the end of Mayearly June 2020, if the draconian measures are continued as of March 20 and March 21.

\subsection{Developing COVID-19 drugs and vaccines}

A modern-day challenge is the proactive management of an Infodemic which grew faster than the actual disease. To avoid and control outbreaks such as the current COVID-19 pandemic, this requires increasing consciousness and sensitization with the proper data. Therefore, by presenting new information and the possibility of disinformation there is a fine balance between constant awareness-raising. In [S67], the authors tackle this gap in this research by developing a lifelong learning framework that provides an accurate knowledge to users in Hindi and English. Using machine learning and natural language processing, it does so by matching sources of confirmed and accurate knowledge, such as the WHO reports against daily news. It provides the narrated material in Hindi through the use of state-of-the-art text to speech engines. Finally, the method enables user feedback to regularly enhance the everyday relevance of news feeds. It demonstrated this method to COVID-19 pandemic containment for Water, Sanitation, Hygiene. Eight human users tested thirteen variations of pre-processing techniques, word embedding, and similarity metrics by measuring the statistics on the agreement. A Cohen's Kappa of 0.54 
achieved the highest performing combination and was implemented as On Alr, WashKaro's Al-powered backend. The authors implemented a new way of tracking communications, deploying the Bluetooth sensors from the smartphone of a person and automatically capturing physical interactions with other users. In addition, the application also includes a symptom self-assessment method focused on WHOapproved criteria, human-cured and validated data to reach out to the community as local language audiovisual material.

In [S68], the authors employed the reverse Vaccinology tool Vaxign as well as the newly designed machine learning tool Vaxign-ML to predict candidates for the COVID-19 vaccine. By analyzing the whole SARS-CoV-2 proteome, six proteins were predicted to be adhesives, including the $S$ protein and five nonstructural proteins (nsp3, 3CL-pro, and nsp8-10), that are essential for viral adherence and host invasion. Vaxign-ML had also predicted the $\mathrm{S}$, nsp3, and nsp8 proteins to induce high defensive antigenicity. The nsp3 protein was not evaluated in any COVID-19 vaccine studies apart from the widely used S protein and was selected for further analysis. In SARS-CoV-2, SARS-CoV, and MERS-CoV, nsp3 was shown to become more conserved than in 15 COVID-19es that infect humans and other animals. The protein had also been predicted to include promiscuous epitopes of MHC-I and MHC-II T-cells, and linear B-cell epitopes located at different protein sites and functional regions. By implementing reverse Vaccinology and machine learning, the authors were predicting possible targets for efficient and healthy production of COVID-19 vaccines. A "Sp / Nsp combination vaccine" including a structural protein(s) (Sp) and a nonstructural protein(s) (Nsp) would then be proposed to induce efficient complementary immune responses.

The authors in [569] conducted drug repurposing analysis using the currently deposited main protease form, 6LU7. For example, pharmacophore- and e-pharmacophore-based hypotheses like AARRH and AARR were established using accessible small molecule inhibitors and used in DrugBank repository screening. Additionally, the hierarchical docking method was introduced with Glide algorithm support. The resulting molecules were then tested with Prime-MM / GBSA algorithm for their binding free energy toward Covid-19 main protease. Significantly, machine learning-based model developed by AutoQSAR algorithm further predicted the resulting antiviral compounds activities. At last, the hit molecules were analyzed via QikProp algorithm for their drug similarity and toxicity criteria. Ultimately, this study generated four possible inhibitors (DB07800, DB08573, DB03744, and DB02986) that are expected to bind with Covid-19's key protease better than commonly used drug molecules like N3 (co-crystallized native ligand), Lopinavir, and Ritonavir.

The work involves the analysis of medicines that are already available on the market and utilized to improve clinical treatment for other diseases, in other words repurpose of existing medications [S70]. The immense nature of drug design and clinical trial procedures also prevents the production of various new formulations of drugs for this infectious disease within a short period. Recently, significant increases in computing capacity combined with advancements in Machine Learning (ML) technology have been used to revolutionize the process of drug production. Correspondingly, a comprehensive analysis using $\mathrm{ML}$ is urgently required to repurpose therapeutic agents. Here this study mentioned that the ML model based on the Naïve Bayes method, which is about 73 \% accurate to predict the drugs that could be utilized for 
COVID-19 treatment. This study predicts about 10 commercial drugs approved by the FDA that can be used to repurpose. I Within all, it suggested that the antiretroviral drug Saquinavir (DrugBank ID-DB01232) is likely to be one of the most successful drugs based on the parameters selected. The results allowed clinical scientists to be more accurate in recognizing and evaluating the COVID-19 therapeutic agents.

In [S71], authors employed an efficient mathematical technique by integrating models based on machine learning $(\mathrm{ML})$ with a high-fidelity set docking simulation to allow the rapid screening of potential therapeutic molecules (or ligands). The screening is designed regarding the binding affinity to either the isolated SARS-CoV-2 S-protein of its host receptor area or the complex Sprotein-human ACE2 interface, potentially limiting and/or disrupting the interactions between the host and virus. Firstly, it applies the proposed screening technique to two drug datasets (CureFFI and DrugCentral) in order to classify hundreds of ligands that bind strongly to the two systems above. All simulations of atom docking were then tested by candidate ligands.

Artificial Intelligence techniques are employed to recognize "progeny" drugs close to those already being evaluated against COVID-19 by the "parents." These methods determine similarity not only by the molecular make-up of the molecules, but also by the "sense" in which particular functional categories are organized and/or by the distribution of pharmacophores in three dimensions. The parent-progeny relationships include drugs of the same indication (mostly antivirals) many of which are in which the "progenies" have specific and possibly less obvious key indications (e.g., immunosuppressive or antiviral parent progenies) [572] The "progenies" are now either accepted drugs or drugs in advanced clinical trials - in the event that the current evaluated "parent" medicines struggle in clinical trials, these "progenies" may then be re-purposed targeting COVID-19 on the time scales applicable to the current pandemic.

\subsection{Managing Healthcare Resources-COVID-19}

In the first 2020, the world started to watch with alarm the spread of a new COVID-19-related pneumonia outbreak, named severe acute respiratory syndrome COVID-19 2 (SARS-CoV-2), which occurred in Wuhan City, Hubei Province, China in late December 2019. Therefore, in [S73], authors conducted a study to assess the spread of the disease and to evaluate the cases of the infected population in need of immediate hospitalization in order to provide appropriate public health services. To that end, a Deep Neural Network (DNN) -based learning tool was utilized to predict COVID-19 infection and the urgent need for hospitalization in some of the infected individuals. It linked the available public hospital resources and determined the need to improve them after the potential significant increase effected by SARS-CoV-2 by provinces in the Andalusian regions, Spain.

\section{Discussion And Future Research Direction.}

Although studies of applying Al techniques for containment COVID-19 have been able to (1) provide a good start to suggest solutions in containing the COVID-19 epidemic, (2) strengthen our understanding of 
the COVID-19 epidemic and how it has spread, many of these studies have been viewed as providing biased, incomplete and poor trustworthiness evidence of the proposed Al techniques for containing COVID-19 pandemic. The analysis results of the systematic review revealed that studies of applying AI techniques for containment COVID-19 are undergoing a crisis that is visible through deficiencies, namely (1) small size of the dataset (2) specific context use (3) lack of giving explicit feedback (4) focus on specific types of Al techniques. Therefore, this study concludes with a set of suggestions that converge on the idea that new studies of applying Al for containment COVID-19:

1. Should use large size of dataset.

Table 4 presents all datasets that used in the reviewed papers, along with its use, size, the country/countries from which it was collected, and source of the dataset (see Appendix B). It is evident from Table 3 and Table 4 that the majority of the studies reviewed (1) used small dataset size, (2) used early-stage datasets, (3) most of the datasets used are Chinese datasets, and (4) neglecting the validation of the dataset. Small dataset is not sufficient to train Al techniques. This is because the performance of the most Al techniques is enhanced on large datasets. Although many Al techniques have proven its applicability for contain COVID-19 epidemics, there are doubts about the accuracy of its results. This weakens their applicability in real life for offering concrete diagnostic and treatment options, as relying on their results can affect patients' lives. Therefore. There is an urgent need to develop large international datasets about COVID-19 pandemic. It is recommended to categorized datasets under specific context. For example, in this study (See Table 4 and Appendix B) we have identified 76 sources of datasets about COVID-19 pandemic which distributed as follows:

- Nineteen sources of dataset for COVID-19 outbreak

- Nine sources of dataset for predicting criticality of COVID-19

- Eleven sources of dataset for distinguishes COVID-19 from chest X-ray.

- Ten sources of dataset for distinguishes COVID-19 from chest CT

- Six sources of dataset for distinguishes COVID-19 from clinical

- Two sources of dataset for distinguishes COVID-19 from genomic

- One source dataset for distinguishes COVID-19 from sound

- Three sources of dataset for detecting and tracking COVID-19

- Twelve sources of dataset for developing COVID-19 drugs and

- Three sources of dataset for managing healthcare resources-COVID-19

These datasets should be updated continuously. In addition, in order to generate a new and comprehensive dataset about COVID-19, data mining research is recommended to discover the pattern of these datasets. Also, the validation of these datasets is necessary to ensure their validity and safety for being used by Al techniques for containment COVID-19 pandemic. Perhaps it is better to classify these datasets by a country which it was collected from. This is important to study whether there is a difference in the results of the application of these datasets from one country to another, which in turn can help in a 
deeper understanding of COVID-19 disease by country and the ability of its residents to respond to this disease.

\section{Should develop hybrid Al systems}

As shown in Table 3 most applied Al algorithms and techniques are related to machine learning. For example, Neural Network and Deep Learning algorithms are the most two techniques applied for containment COVID-19 pandemic. As many of the reviewed studies reported that small dataset is the main limitation of their studies, to use these datasets to train and test such algorithms will lead to decrease their performance and inaccuracy of their results. Even the machine learning algorithms are powerful predictive algorithms which can be used to deal with more complex structure of data, but they need large dataset, training for a long time, and they have poor interpretability (LeCun et al., 2015). Which in turn creates a conflict for the adoption of these algorithms. However, due to fact that no single Al techniques outperforms all other techniques. Future research should give more attention to hybrid $\mathrm{Al}$ systems that combined new trends of Al techniques (e.g., metaheuristic algorithm) to benefit from the different characteristics of different algorithms. For example, but not limited to, salp swarm algorithm (Abualigah et al., 2019), moth-flame optimization (Shehab et al.,2019), and cuckoo search algorithm (Shehab et al.,2017) are a very promising and interesting algorithm that has already been successfully applied to several problems in medical research area due to their contain simplicity, speed in searching, and simple hybridization with other algorithms.

\section{Should not focus on a specific research area over another}

Figure 2. indicates that $45 \%$ of Al techniques were specifically designed to diagnosing COVID- 19 cases (e.g., 18\% distinguishes COVID-19 from chest X-ray, 14\% distinguishes COVID-19 from chest CT images, $8 \%$ distinguishes COVID-19 from clinical test, $3 \%$ distinguishes COVID-19 from genomic signatures, $2 \%$ distinguishes COVID-19 from sound signal). $38 \%$ of Al techniques were developed to address COVID-19 prediction cases (e.g., $26 \%$ for COVID-19 outbreak prediction and $12 \%$ in predicting criticality of COVID-19 cases). While, $8 \%$ of Al techniques were specifically implemented for detecting and tracking COVID-19 cases. Also, $8 \%$ of Al techniques were used for developing COVID-19 drugs and vaccines. Finally, $1 \%$ of $\mathrm{Al}$ technique used for managing healthcare resources-COVID-19.

The majority of reviewed Al techniques were developed for prediction and diagnosing COVID- 19 pandemic neglecting other important area such as developing COVID-19 drugs, managing healthcare resources, and detecting and tracking COVID-19 cases. Therefore, future research must take into account that developing Al techniques for these areas is no less important than others and is essential in containing the COVID-19 pandemic. For example, proposing Al techniques that aim to produce drugs and medicines to treat infected COVID-19 patients and Al techniques to investigate vaccines to prevent the virus of COVID-19 is highly recommended and persistent need. In addition, developing Al techniques for managing healthcare resources in recommended. This is because the numbers of people infected with the COVID-19 are large, widespread, and is constantly increasing, hence the management of healthcare 
resources is necessary to ensure the continuity of the healthcare process without interruption or congestion.

As presented in Table 4 and Figure 3, the majority of reviewed studies are conducted to containment COVID-19 pandemic in China (e.g., 19 studies). Following by 13 studies conducted to contain the COVID19 pandemic in worldwide. While, USA and Italy produced 5 studies for each. Following by India 4 studies. This pandemic is not limited to a single country. It is a global pandemic. Therefore, it is necessary for future research to take into account the developing countries (e.g., African countries, middle eastern countries, Latin American countries, etc.) These countries are already suffering from a scarcity of health resources and crisis management, Al techniques for containment COVID-19 can help them in making the right decisions in managing the crisis in various level health, economically and socially.

\section{Conclusion}

In this paper the applications of Al techniques for containing COVID-19 were reviewed. The main contribution of this systematic literature review was to introduce new lines of inquiry that could inspire researchers to conduct further researches or improve existing studies within the field applying Al techniques for containing COVID-19 pandemic. Seventy four research papers were identified, retrieved, summarized and analyzed. The analysis result revealed that this area of using Al techniques for containing COVID-19 pandemic still in the infant stage and is not yet complete. Research in such area is undergoing a crisis that is made visible by the numerous problems and deficiencies. These deficiencies emphasize the importance of rethinking the proposed Al techniques, datasets, and research focus area for containing COVID-19 pandemic. To be accepted in the real world, these studies should increase the performance and the result accuracy of applied Al techniques by using large size of dataset, develop hybrid Al systems. Also, they should increase the generalizability of the proposed studies as the COVID-19 disease is a global pandemic. However, Al techniques are still promising, interesting and more and more becoming mature which make them more trustable and attractive to help in containing COVID-19 pandemic.

\section{Declarations}

The authors have declared no competing interest.

\section{References}

Abualigah, L., Shehab, M., Alshinwan, M. and Alabool, H., 2019. Salp swarm algorithm: a comprehensive survey. Neural Computing and Applications, pp.1-21.

Alimadadi, A., Aryal, S., Manandhar, I., Munroe, P.B., Joe, B. and Cheng, X., 2020. Artificial Intelligence and Machine Learning to Fight COVID-19. Physiol Genomics, doi:10.1152/physiolgenomics.00029.2020 
Bullock, J., Pham, K.H., Lam, C.S.N. and Luengo-Oroz, M., 2020. Mapping the landscape of artificial intelligence applications against COVID-19. arXiv preprint arXiv:2003.11336.

Coronavirus disease (COVID-2019) situation reports: 436

http://www.who.int/emergencies/diseases/novel- coronavirus-2019/situation-reports.

Fuchs, C., 2020. Everyday Life and Everyday Communication in Coronavirus Capitalism. tripleC: Communication, Capitalism \& Critique. Open Access Journal for a Global Sustainable Information Society, 18(1), pp.375-399.

https://www.who.int/emergencies/diseases/novel-coronavirus-2019

https://www.worldometers.info/coronavirus/

Ilyas, M., Rehman, H. and Nait-ali, A., 2020. Detection of Covid-19 From Chest X-ray Images Using Artificial Intelligence: An Early Review. arXiv preprint arXiv:2004.05436

Keele, S., 2007. Guidelines for performing systematic literature reviews in software engineering. In Technical report, Ver. 2.3 EBSE Technical Report. EBSE. sn.

Kitchenham, B., Brereton, O.P., Budgen, D., Turner, M., Bailey, J. and Linkman, S., 2009. Systematic literature reviews in software engineering-a systematic literature review. Information and software technology, 51(1), pp.7-15.

Latif, S., Usman, M., Manzoor, S., Iqbal, W., Qadir, J., Tyson, G., Castro, I., Razi, A., Boulos, M.N.K. and Crowcroft10, J., Leveraging Data Science To Combat COVID-19: A Comprehensive Review.

LeCun, Y., Bengio, Y. and Hinton, G., 2015. Deep learning. nature, 521(7553), pp.436-444. Naudé, W., 2020. Artificial Intelligence against COVID-19: An Early Review.

Nguyen, T.T., 2020. Artificial Intelligence in the Battle against Coronavirus (COVID-19): A Survey and Future Research Directions. Preprint, DOI: 10.13140/RG.2.2.36491.23846

Rahmatizadeh, S., Valizadeh-Haghi, S. and Dabbagh, A., 2020. The role of Artificial Intelligence in Management of Critical COVID-19 patients. Journal of Cellular \& Molecular Anesthesia, 5(1), pp.16-22.

Shehab, M., Abualigah, L., Al Hamad, H., Alabool, H., Alshinwan, M. and Khasawneh, A.M., 2019. Mothflame optimization algorithm: variants and applications. Neural Computing and Applications, pp.1-26.

Shehab, M., Khader, A.T. and Al-Betar, M.A., 2017. A survey on applications and variants of the cuckoo search algorithm. Applied Soft Computing, 61, pp.1041-1059.

Shi, F., Wang, J., Shi, J., Wu, Z., Wang, Q., Tang, Z., He, K., Shi, Y. and Shen, D., 2020. Review of artificial intelligence techniques in imaging data acquisition, segmentation and diagnosis for covid-19. IEEE Reviews in Biomedical Engineering. 
Vaishya, R., Javaid, M., Khan, I.H. and Haleem, A., 2020. Artificial Intelligence (AI) applications for COVID19 pandemic. Diabetes \& Metabolic Syndrome: Clinical Research \& Reviews.

van der Schaar, M. and Alaa, A., 2020. How artificial intelligence and machine learning can help healthcare systems respond to COVID-19.

Wynants, L., Van Calster, B., Bonten, M.M., Collins, G.S., Debray, T.P., De Vos, M., Haller, M.C., Heinze, G., Moons, K.G., Riley, R.D. and Schuit, E., 2020. Prediction models for diagnosis and prognosis of covid-19 infection: systematic review and critical appraisal. bmj, 369.

\section{Tables}

Due to technical limitations, Tables 3 and 4 are only available as a download in the supplemental files section

\section{Figures}

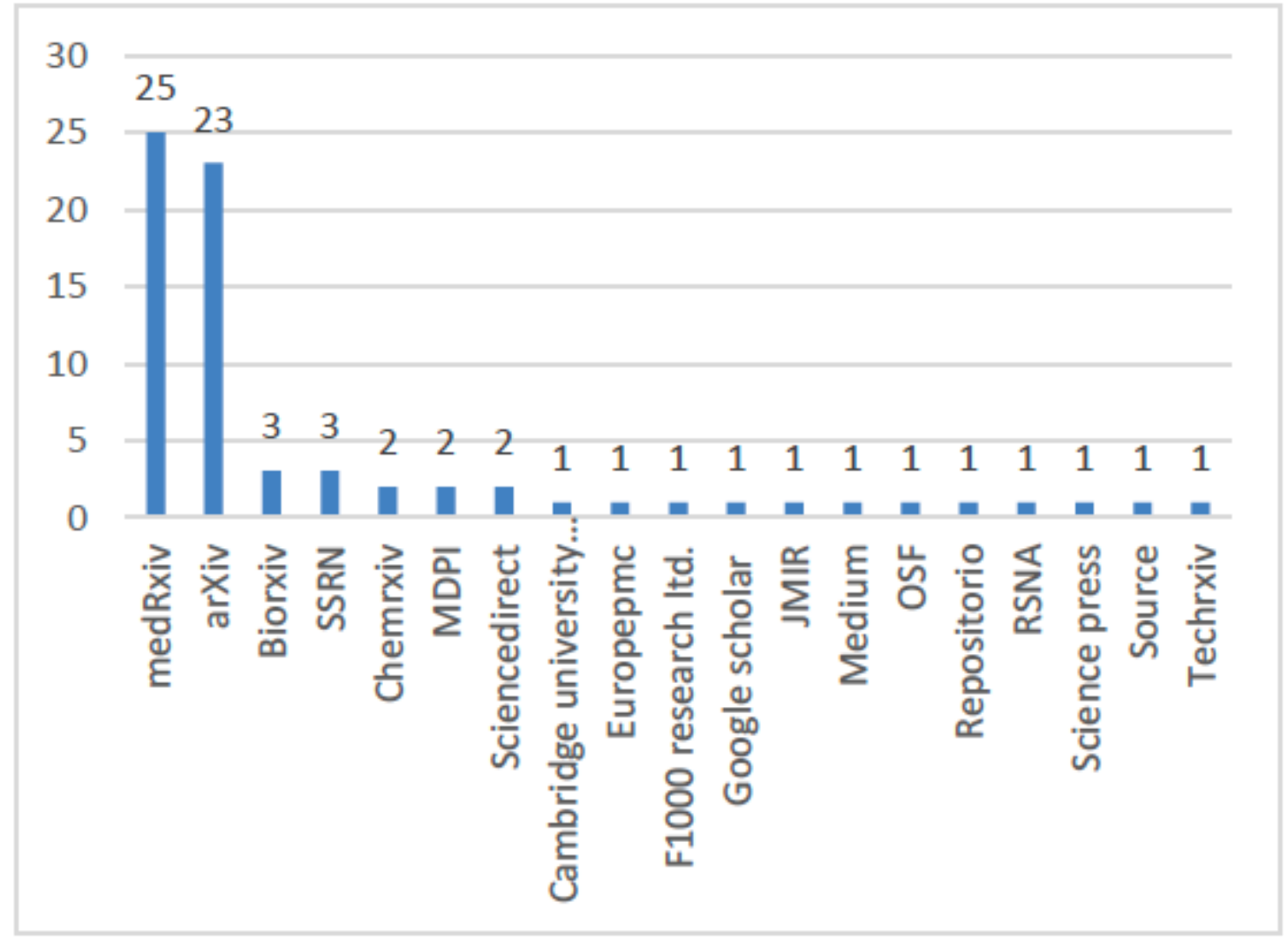

Figure 1

Distribution of the selected papers 


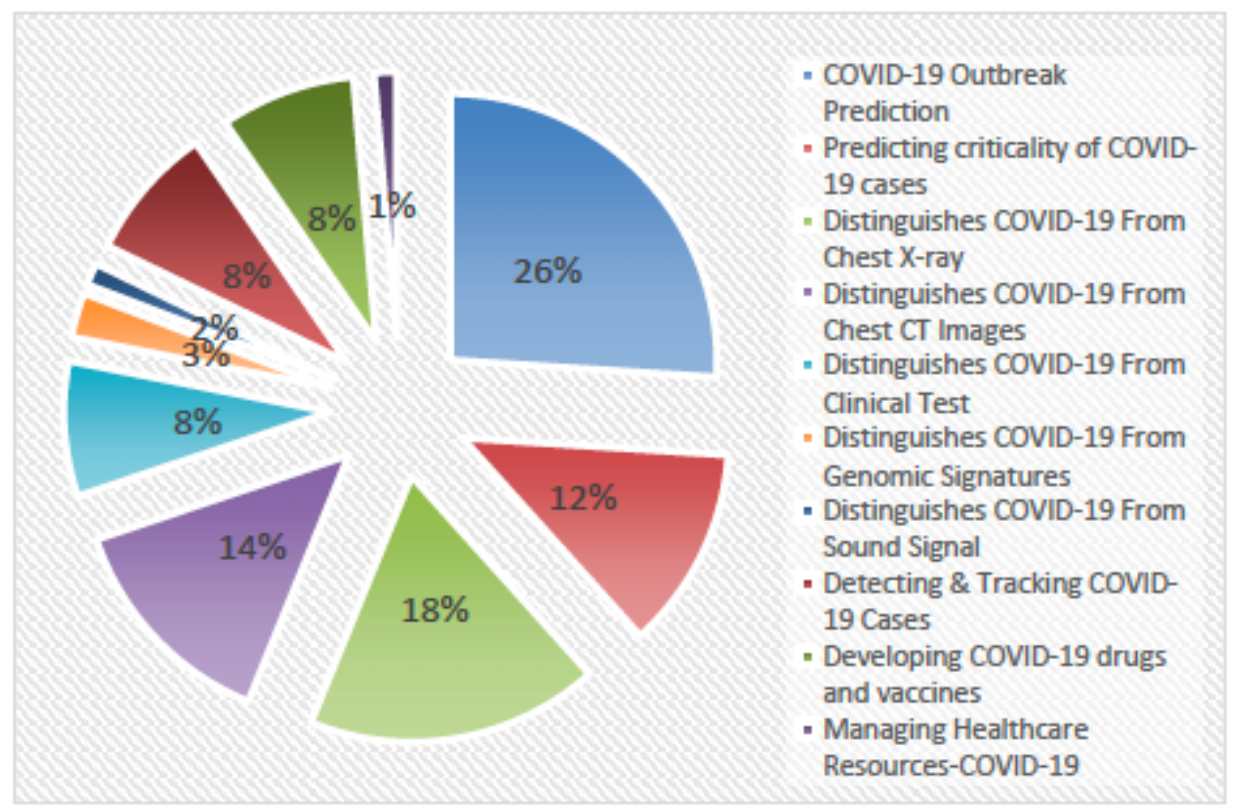

Figure 2

Al techniques Application for COVID-19

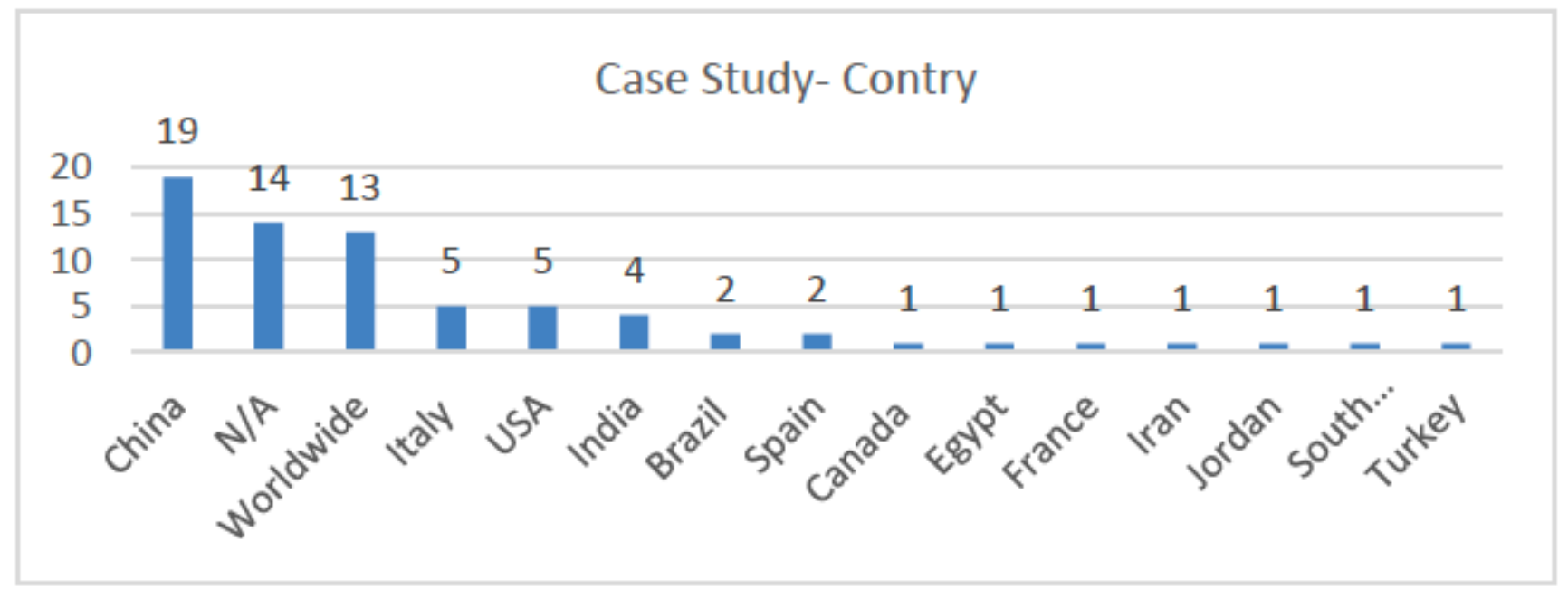

Figure 3

Al techniques Application- country case study

\section{Supplementary Files}

This is a list of supplementary files associated with this preprint. Click to download.

- Appendices.pdf

- Tables3and4.pdf 